\title{
Reflexions on a newly discovered diabetogenic gene, wolframin (WFS1)
}

\author{
K.-D. Gerbitz \\ Diabetes Research Group and Institute of Clinical Chemistry, Molecular Diagnostics and Mitochondrial Genetics, Academic \\ Hospital Schwabing, Munich, Germany
}

\begin{abstract}
Albrecht von Graefe [1], a German ophthalmologist, was the first who described an association between diabetes mellitus and visual disturbance, 140 years ago in 1858. Then 80 years later Wolfram and Wagener [2] characterized four patients with a syndrome consisting of diabetes mellitus and optic atrophy which was called Wolfram syndrome thereafter. The acronym DIDMOAD describes patients with diabetes insipidus and deafness as additional symptoms (OMIM No222300). Among these symptoms early onset insulin-dependent diabetes mellitus and optic atrophy have been taken as the first and essential features of the syndrome. Atonic bladder, ataxia, hypogonadism, insomnia, and seizures are additional but less frequent neurological and endocrinological abnormalities whereas a high incidence of depression and psychotic behaviour is attributed to the syndrome [3]. Wolfram syndrome has been considered an autosomal recessive disorder with consanguinity in about $25 \%$ of patients being the strongest evidence of this mode of inheritance. In $1993 \mathrm{Bu}$ and Rotter hypothesized [4] that the syndrome could be a mitochondrial-mediated disorder because most of the phenotypes characteristic of the syndrome are consistent with an ATP supply defect. Thus, the agglomeration of these clinical symptoms is the strongest argument for a mitochondrial origin of the disease. In 1994 the syndrome was mapped to the short arm of chromosome 4 [5]. Using different techniques, most recently, cooperating Japanese and American groups [6] and our group in Munich [7] succeeded in discovering the gene, which they named WFS1 (Wolfram syndrome 1) and we wolframin gene.
\end{abstract}

Corresponding author: K.-D. Gerbitz, Diabetes Research Group and Institute of Clinical Chemistry, Molecular Diagnostics and Mitochondrial Genetics, Academic Hospital Schwabing, D-80804 Munich, Germany
The wolframin gene is composed of seven small exons and one large exon of $1812 \mathrm{bp}$, spanning about $33.4 \mathrm{~kb}$ of genomic DNA. No cross-hybridization is observed with genomic DNA from yeast and Drosophila, but a mouse homologue with $83 \%$ sequence identity does exist. The gene encodes wolframin, a 890 amino acid-long protein, corresponding to a predicted molecular weight of almost $100 \mathrm{kD}[6,7]$. It shows no perceptible homology to published DNA or protein sequences. Structurally it is characterized by a central hydrophobic domain comprising, at least, nine helical transmembrane segments flanked on both sides by hydrophilic domains and is, therefore, identified as a transmembrane protein $[6,7]$. The physiological function of this novel protein is totally unclear.

In $1993 \mathrm{Bu}$ and Rotter [4] stated that "the identification of the nuclear mutations in Wolfram syndrome will provide an important candidate gene for the study ... of diabetes" Now, we are at this point; what did we learn and how shall we proceed as the situation seems to offer more questions than answers?

The most important answer is striking: there is strong evidence that the wolframin gene is, indeed, involved in Wolfram syndrome. The mutation screening in 18 Wolfram families by both groups $[6,7]$ showed a series of different mutations, including stop, frameshift, deletion and insertion mutations mostly located in exon 8 . Thus, wolframin gene loss of function mutations is the cause of the Wolfram syndrome.

Some of the most important questions are outlined in the following.

Is Wolfram syndrome a homogenous disease entity? At present there is no conclusive answer to this question with all its various facettes. Tentatively, it is supposed the syndrome is a disorder of two genomes. 
Apart from the chromosomal wolframin (WSF1) mutations discovered recently $[6,7]$ several authors have described point [8] and length mutations [911] of the mitochondrial DNA (mtDNA). In contrast, no causative mtDNA length mutations (deletions, insertions) were found in any of our 12 patients with the syndrome regardless of whether the patient carried wolframin mutations or not [12]. The same held true when we screened our Wolfram patients for a series of mtDNA point mutations usually attributed to typical mitochondrial disorders like MELAS (mitochondrial encephalopathy with lactic acidosis and stroke-like episodes) and LHON (Leber hereditary optic neuropathy) [13]. A mitochondrial tRNA screen in Wolfram patients had already been done by others [14] without any positive disease linkage. Moreover, we were given the opportunity to look for wolframin mutations in patients described by others [9-11]. In the French patient [9] no wolframin (WFS1) mutations could be found. Thus, this patient with a $7.4 \mathrm{~kb}$ deletion and suffering from some phenotypic symptoms not typical for Wolfram syndrome (for instance, chronic progressive external ophthalmoplegia) does not represent either the classical Wolfram syndrome or a heterogeneous subtype of the syndrome. In some Spanish patients we found wolframin mutations but in others we did not. Furthermore, all patients from six Wolfram families described by Inoue et al.[6] carried different WSF1 (wolframin) mutations, four were homozygous, and two were compound heterozygous. In contrast, we detected homozygous wolframin mutations only in 5 of our 12 patients. Possible pathogenic consequences of heterozygous missense or stop mutations as well as homozygous in-frame mutations observed in four of our patients are still to be investigated. In three of our Wolfram families no mutations in either of the two alleles could, however, be detected [7]. There are several possibilities to explain this: for instance, mutations in exon 1 , which was not included in our mutation screen, or in the regulatory flanking regions of the gene or intronic deletions could be of pathogenic relevance. Non-allelic heterogeneity could be an alternative explanation [15]. Moreover, a recent publication [16] suggests the possibility of a further nuclear gene locus associated with Wolfram syndrome. In the Wolfram families investigated by our group no obvious genotype-phenotype relation emerged [7], regardless of whether patients carried wolframin mutations or not. There was also no relation between the type of wolframin mutations and the mtDNA haplogroup to which the patients belonged [13]. Thus, at present, the question of whether Wolfram syndrome is a homogeneous or heterogeneous disease remains open pending further studies; the same applies to a possible relation between wolframin mutations and the vulnerability of the mitochondrial genome.
Does heterozygosity of the wolframin gene predispose to illness? Typically, heterozygous carriers of genes for autosomal recessive syndromes do not have any of the identifiable physical findings of the syndrome seen in homozygotes [17]. It has, however, been proposed that Wolfram heterozygotes, are predisposed to psychiatric illness [17]. The description of wolframin mutations now offers the possibility of testing these assumptions directly.

Is Wolfram syndrome a mitochondrial disorder - in other words - is wolframin a mitochondrial protein? Up to now there is no definite answer to this question since nothing is known either about the intracellular location of the protein or its physiological function. From northern blots $[6,7]$ it can be assumed that wolframin is expressed ubiquiteously in most, if not all organs, but its expression pattern does not seem to correlate with the number of mitochondria typically existing in the respective organs. In our study [7] northern blot signals were strong in heart, intermediate in brain, placenta, lung and pancreas and weak in liver, kidney and skeletal muscle, the latter a tissue with a high number of mitochondria. This does not necessarily argue, however, against a mitochondrial location of wolframin, since it is known that expression levels of genes encoding mitochondrial proteins do not, in any case, reflect the mere number of mitochondria in such organs. Histological and biochemical investigations in skeletal muscles from Wolfram patients were reported only in a few cases. In one subject patches of dense peripheral staining due to increased numbers of sub-sarcolemnal mitochondria were shown, although no true ragged-red fibres were seen [18]. This patient also had an appreciable deficiency of glutamate dehydrogenase in the skeletal muscle. A further patient was reported [19] with impaired respiratory chain complex I activity (NADH oxidoreductase) and partial deficiency of glutamate dehydrogenase. In the skeletal muscle of one of our Wolfram patients a few ragged-red fibres were found [7] indicating an attempt to compensate for an impaired mitochondrial energy production. Moreover, wolframin does not contain a typical mitochondrial leader sequence indicating mitochondrial targeting. Again, this is not a strong argument against a mitochondrial location of wolframin since not all mitochondrially targeted proteins carry such a leader sequence. The development of specific antibodies will clarify the intracellular location of wolframin in the near future.

Is there a selective beta-cell death in Wolfram syndrome? A report in 1989 [20] described a high concordance rate for the age of onset of diabetes mellitus among siblings from Wolfram families with multiple cases of the syndrome when compared with patients with classical autoimmune Type I (insulin-depen- 
dent) diabetes mellitus. This together with the lack of markers for an autoimmune process is evidence that Wolfram syndrome results from a genetically programmed selective beta-cell death and not from a functional defect in structurally intact islets. An excess of juvenile-onset diabetes mellitus in siblings of affected Wolfram patients was also reported by others [21]. Histological examination [20] showed strongly atrophic islets, staining for insulin almost no insulin-containing cells and for glucagon, somatostatin and pancreatic polypeptide scattered positive cells indicating a selective loss of beta cells and an apparent preservation of alpha and delta cells. This first report on a selective loss of beta cells in Wolfram syndrome was confirmed some years later in 9 of 11 patients with Wolfram syndrome [22]. Conflicting results were, however, published on peripheral C-peptide concentrations in Wolfram patients, ranging from near normal concentrations of insulin to severe deficiency but the nationwide study of Wolfram syndrome in the United Kingdom found low C-peptide concentrations and thus insulin deficiency in all ten patients investigated [23].

Is there any evidence for an autoimmune process in Wolfram syndrome? The answer is clearly no. None of the respective studies have shown either an association to the HLA system or any humoral immune response like the occurrence of Type I diabetic specific antibodies such as islet cell or glutamic acid decarboxylase antibodies in patients with Wolfram syndrome [23].

What is the reason for the evidence that Wolfram patients suffer to a much lower degree from specific diabetic late complications than patients with classical autoimmune Type I diabetes? The thorough study on Wolfram syndrome in the United Kingdom found a lack of retinopathy and nephropathy in a series of patients with a median duration of diabetes mellitus of 24 years, despite poor glycaemic control [23]. Similar results came from other groups [21]. The reason for this surprising fact is not known but speculations are wide ranging and there are some interesting research projects. For instance, the development of specific diabetic late complications might be mainly under immune-genetic control and thus not exclusively a matter of glycaemic control by therapy.

Does the morphological phenotype in Wolfram syndrome give any hints on the physiological function of wolframin? Atrophy and death of specific cell systems are the most common morphological hallmarks in Wolfram syndrome. Neuropathological studies showed atrophy of the optic nerves and chiasm, of the olfactory bulbs and tracts, loss of neurons in the lateral geniculate nuclei, loss of fibres in the cochlear nerve, olivopontocerebellar atrophy and demyelina- tion of the pyramidal tracts as the main neuropathological findings [24]. On the other hand, it is obvious that endocrine deficiencies are an essential finding in all patients with the syndrome. Thus, both systems (the neuronal network and the endocrinium) are affected in Wolfram syndrome, although both seem to be of different embryological origins. At least, for beta cells it is, however, well established nowadays that they share a large number of similarities with neuronal cells [25]. Proteins like glutamic acid decarboxylase, tyrosine and dopamine- $\beta$-hydroxylase, receptors for glutamate, gamma-butyric acid (GABA) and neurotrophin, neurofilament proteins, thyrotropin releasing hormone, synapsin I and many other proteins have been shown to be expressed in both beta and neuronal cells. Furthermore, the presence or absence of specific transcriptional activators and repressors which regulate the expression of a large battery of neuron-specific genes have been described in both cell types [25]. As, both cell types are members of the family of excitable tissues it seems probable that wolframin functions in a process protecting these cells from programmed cell death.

The chance to study a gene responsible for maintenance of specialized neuronal and endocrine cells, like beta cells, should be of great help to clear up the mechanisms underlying various neurodegenerative disorders, not only diabetes mellitus.

\section{References}

1. von Gräfe A (1858) Über die mit Diabetes vorkommenden Sehstörungen. Arch Ophthalmol 4: 230-234

2. Wolfram DJ, Wagener HP (1938) Diabetes mellitus and simple optic atrophy among siblings: report of four cases. Mayo Clin Proc 13: 715-718

3. Swift RG, Sadler D, Swift M (1990) Psychiatric findings in Wolfram syndrome homozygotes. Lancet 336: 667-669

4. Bu X, Rotter JI (1993) Wolfram syndrome: a mitochondrial-mediated disorder? Lancet 342: 598-600

5. Polymeropoulos MH, Swift RG, Swift M (1994) Linkage of the gene for Wolfram syndrome to markers of the short arm of chromosome 4. Nat Gen 8: 95-97

6. Inoue H, Tanizawa Y, Wasson J et al. (1998) A gene encoding a transmembrane protein is mutated in patients with diabetes mellitus and optic atrophy. Nat Gen 20: 143-148

7. Strom T, Hörtnagel K, Hofmann S et al. (1998) Diabetes insipidus, diabetes mellitus, optic atrophy and deafness (DIDMOAD) caused by mutations in a novel gene (wolframin) coding for a predicted transmembrane protein. Hum Mol Genet 7: 2021-2028

8. Pilz D, Quarrel OWJ, Jonas EW (1993) Mitochondrial mutation commonly associated with Leber's hereditary optic neuropathy observed in a patient with Wolfram syndrome (DIDMOAD). J Med Genet 31: 328-330

9. Rötig A, Cormier V, Chatelain P et al. (1993) Deletion of mitochondrial DNA in a case of early onset diabetes mellitus, optic atrophy and deafness (Wolfram syndrome, MIM 222300). J Clin Invest 91: 1095-1098

10. Barrientos A, Volpini V, Casademont J et al. (1996) A nuclear defect in the 4 p16 region predisposes to multiple mi- 
tochondrial DNA in families with Wolfram syndrome. J Clin Invest 97: 1570-1576

11. Barrientos A, Casademont J, Saiz A et al. (1996) Autosomal recessive Wolfram syndrome associated with an $8.5 \mathrm{~kb}$ mtDNA single deletion. Am J Hum Genet 58: 963-970

12. Hofmann S, Bezold R, Jaksch M et al. (1997) Wolfram (DIDMOAD) syndrome and Leber hereditary optic neuropathy (LHON) are associated with distinct mitochondrial haplotypes. Genomics 39: 8-18

13. Hofmann S, Jaksch M, Bezold R et al. (1997) Population genetics and disease susceptibility: characterization of central European haplogroups by mtDNA gene mutations, correlation with D loop variants and association with disease. Hum Mol Genet 6: 1835-1846

14. Van den Ouweland JMW, Bruining GL, Lindhout D, Wit J-M, Veldhuyzen FE, Maassen JA (1992) Mutations in mitochondrial tRNA genes: non-linkage with syndromes of Wolfram and chronic progressive external ophthalmoplegia. Nucleic Acids Res 20: 679-682

15. Collier DA, Barrett TG, Curtis D et al.(1996) Linkage of Wolfram syndrome to chromosome 4p16.1 and evidence for heterogeneity. Am J Hum Genet 59: 855-863

16. El-Shanti H, Ailouni K, Jarrah N, Druhan LJ, Lidral AC (1998) Clinical and genetic heterogeneity of Wolfram syndrome. Am J Hum Genet 63: A103 (Abstract 567)

17. Swift RG, Polymeropoulos MH, Torres R, Swift M (1998) Predisposition of Wolfram syndrome heterozygotes to psychiatric illness. Mol Psychiatr 3: 86-91
18. Bundey S, Poulton K, Whitwell H, Curtis E, Brown IAR, Fielder AR (1992) Mitochondrial abnormalities in the DIDMOAD syndrome. J Inherit Metab Dis 15: 315-319

19. Bundey S, Fielder A, Poulton K (1993) Letter to Lancet 342: 1059-1060

20. Karasik A, O'Hara C, Srikanta S et al. (1989) Genetically programmed selective islet $\beta$-cell loss in diabetic subjects with Wolfram syndrome. Diabetes Care 12: 135-138

21. Fraser F, Gunn T (1995) Diabetes mellitus, diabetes insipidus and optic atrophy: an autosomal recessive syndrome? J Med Genet 14: 190-193

22. Kinsley BT, Dumont RH, Swift M, Swift RG (1995) Morbidity and mortality in the Wolfram syndrome. Diabetes Care 18: 1566-1570

23. Barrett TG, Bundey SE, Macleod AF (1995) Neurodegeneration and diabetes: UK nationwide study of Wolfram (DIDMOAD) syndrome. Lancet 346: 1458-1463

24. Genis D, Davalos A, Molins A, Ferrer I (1997) Wolfram syndrome: a neuropathological study. Acta Neuropathol (Berl) 93: 426-429

25. Atouf F, Czernichow P, Scharfmann R. (1997) Expression of neuronal traits in pancreatic beta cells. J Biol Chem 272: 1929-1934 